\section{Anesthetic management in awake craniotomy}

\author{
FRANCESA ZORZI • MASSIMILIANO SALTARINI • PAOLO BONASSIN • \\ MARCO VECIL • PALMA DE ANGELIS • AMATO DE MONTE
}

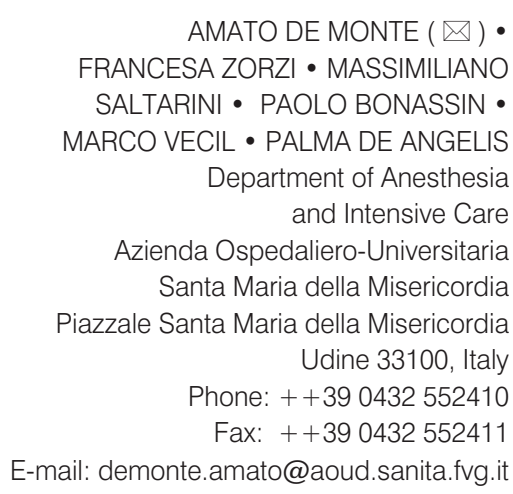

AMATO DE MONTE $(\bowtie) \bullet$ FRANCESA ZORZI • MASSIMILIANO

SALTARINI • PAOLO BONASSIN • MARCO VECIL • PALMA DE ANGELIS Department of Anesthesia and Intensive Care

Azienda Ospedaliero-Universitaria

Santa Maria della Misericordia

Piazzale Santa Maria della Misericordia Udine 33100, Italy

Phone: ++390432552410

Fax: + +390432 552411

E-mail: demonte.amato@aoud.sanita.fvg.it

\begin{abstract}
Resection of brain tumors may cause neurological sequelae, according to the site and size of the brain tissue removed. Awake craniotomy has been proposed as a surgical approach to satisfy criteria of radical surgery while minimizing eloquent brain damage.

The most critical aspect of awake craniotomy is to maintain adequate patient comfort, analgesia, immobility and cooperation during a long surgical operation, ensuring in the meanwhile the safety, control and maintenance of vital functions.

Apart from pharmacological, surgical, technical knowledge and skillfulness, the ability to maintain close psycho-emotional contact and support with the patient throughout the operation is a fundamental task that the anesthesiologist has to pursue for the operation to be successfully managed.
\end{abstract}

This review summarizes the current opinion on anesthetic management of patients undergoing awake craniotomy.

Key Words: anesthesia, awake craniotomy, brain mapping, brain tumor, neuro-oncology

\section{Introduction}

The main challenge of oncological surgery is the radical removal of a tumor. A general assertion states that the larger the resection the lower the risk of recurrence of the lesion and the higher the chance of the patient's survival. But an extensive tissue excision may favor the occurrence of an unpredictable degree of functio laesa, depending on the location of the malignancy. Particularly in neuro-oncology, the neurological sequelae due to tumor excision may cause severe disability compromising the patient's social life. Therefore, the aim to remove the maximum amount of lesion without impairing neurological function, has pushed the physicians and industry to develop sophisticated surgical approaches to be performed in awake and responding patients, so as to evaluate neurological dysfunction before tissue removal.(1)
Awake craniotomy dates back to the second half of the $19^{\text {th }}$ century, and at that time, the indication was epilepsy surgery performed under local anesthesia. Subsequently, this surgical practice has been extended also to the resection of tumors involving the functional cortex and finally, in more recent years, the indications have further extended to include the removal of supratentorial tumors, regardless of the involvement of the cortex. (2)

Craniotomy in awake patients has evolved and extended its indication as a direct consequence of the following driving forces: the huge improvement of diagnostic tools; the impressive development of intraoperative functional neurosurgical technology; the enhancement of anesthesia monitoring devices, the pharmacokinetic and pharmacodynamic properties of the new anesthetic agents and the modality by which they are delivered to the patients. (1)

The main advantage for the awake neurosurgical approach is to facilitate intraoperative electrocorticography and cortical mapping for the accurate identification of brain areas which control motor function and speech. (3)

Functional magnetic resonance imaging has produced considerable progresses in non-invasive mapping of brain functional areas, allowing very early tumor stratification. However, its employ during surgery is not feasible on a routine basis and intraoperative testing of language and motor function continues to be the gold standard for a radical surgical resection while minimizing eloquent brain damage.

Apart from tumor anatomical location, mandatory prerequisites for awake craniotomy are a fully cooperative patient and optimal collaboration between anesthesia and neurosurgical staff, to realize what is defined as function-controlled neurosurgery. $(4,5)$ Indeed, evolution of general anesthesia in neurosurgery has permitted adequate control of vital parameters, neurological function and intracranial pressure; at the same time these aspects ensure 
optimal working conditions for the neurosurgeon; but intraoperative monitoring of functional lesions of the central nervous system is severely inhibited by general anesthesia: some higher cortical brain functions (i.e. speech) cannot be monitored during surgery.

\section{Anesthesia technique: general statements}

"Awake craniotomy" is a misleading term. Because the different surgical phases require various levels of sedation, the patient is maintained completely awake only during the mapping procedure, which requires patient cooperation, and during the early resection of the tumor.

Providing appropriate sedation and analgesia for awake craniotomy is a challenge for the anesthesiologist. A continuous and rapid modulation of sedation and analgesia level is absolutely necessary to manage painful surgical stimuli and specific neurophysiological responses (cortical stimulation or speech testing). Of the utmost importance, the patient must be awake and alert during brain mapping. (6) In the meantime vital functions have to be ensured, as well as emergency support if deterioration of clinical status should occur.

Several combinations of sedation, analgesia, and anesthetic techniques have been described in the medical literature. The "asleep awake asleep technique", includes an initial phase of general anesthesia, eventually with the insertion of a supraglottic airway device, followed by intraoperative awakening for language mapping during tumor resection, and finally, back again to general anesthesia during the craniotomy closure. Monitored anesthesia care or conscious sedation technique, requires the use of short acting anesthetic agents combined with local anesthesia during the painful phases of surgery. $(7,8)$

Whatever the strategy of anesthesia chosen, the final goals are to allow the neurosurgeon to take advantage of the patientžs cooperation and preserve general homeostasis. The anesthesia technique selected requires optimal analgesia during nociceptive stimulations; sedation, anxiolysis, immobility and comfort during mapping and the resection procedure and, finally, prevention of side effects such as nausea, vomiting and seizures. Maintenance of a patent airway, adequate ventilation, hemodynamic stability and brain relaxation is mandatory. (1)

Awake cranial surgery has specific risks and patients are exposed to increased physical and mental stress. In addition to the classical issues commonly recommended before any type of anesthesia, the anesthesiologist should pay particular attention to other specific points:

- he/she must predict a difficult intubation and detect any risk factor that could favor upper airway obstruction or respiratory depression, such as obesity or sleep apnea syndrome

- when dealing with epileptic patients, he/she should be aware of the type and frequency of seizures as well as the anticonvulsant therapy

- the anesthesiologist should evaluate the degree of intracranial hypertension according to clinical signs and imaging

- hemorrhagic risk of the procedure, depending on type of lesion, clinical history and routine medications have also to be taken into account

- degree of anxiolysis, tolerance to pain and any neurological deficit that could impede the patient's cooperation during surgery, are crucial factors to be accurately considered too.

An essential feature of patient information during preoperative assessment is an accurate and detailed explanation of the procedure (i.e. intraoperative sounds of surgical instruments, head immobilization, voices...). The patient must be informed regarding the sequence of events occurring during surgery as well as the possible complications, in order to minimize fear about surgery and anesthesia. Obtaining the patient's confidence and agreement for cooperation during surgery is a fundamental factor for a successful procedure. $(1,9)$ Specific problems such as agitation, restlessness and lack of co-operation, may become a serious concern during awake brain surgery.

It is imperative that candidates for an awake craniotomy are psychologically prepared to be actively involved in the procedure: the cognitive level and motivation of the patient is a key to success!

Many of the early reported anesthetic protocols concerning awake-asleepawake techniques that often require elaborate maneuvers of intraoperative airway management, or relatively deep intravenous sedation with drugs that have a slow offset of action. Sarang and Dinsmore retrospectively examined three different anesthesia techniques: sedation with propofol, fentanyl, droperidol and midazolam; asleepawake-asleep with propofol infusion, i.v. fentanyl and spontaneous ventilation via a LMA; an asleep-awake-asleep anesthesia with propofol and remifentani and IPPV via a LMA. All these procedures guarantee reasonable patient comfort and satisfaction, but the deep sedation they produce, can jeopardize the benefits of the functional intraoperative testing. (7)

Nowadays, propofol is the first choice hypnotic; it can be administrated using a target control infusion technique.

Propofol associated with remifentanil, which has a very short half life, causes a rapid and fine modulation of sedation depth; furthermore an accurate titration of both drugs allows avoidance of devices for airways control. $(10,11)$

The techniques described for more secure airway protection include endotracheal intubation and awakening without endotracheal tube withdrawal (disallowing vocalization testing); extubation and re-intubation after testing; placement, removal and replacement of a LMA. $(7,12)$ Non-invasive positive pressure ventilation has also been described. (13)

Alfa 2 agonists clonidine is often used as an analgesic co-adjuvant. Dexmetedomidine appears to be of increasing interest because it seems to provide sedation closer to natural sleep, it gives anxiolysis and analgesia, decreases 
the need for opioids and antihypertensive drugs and it does not interfere with respiratory function. $(14,15,16)$ Unfortunately, at the moment it is not yet available in most European countries.

\section{Airway management}

Airway care is a major challenge during surgery in awake craniotomy, and respiratory complications are the most feared, especially during an unprotected airway sedation technique. Whichever technique of awake craniotomy used, it is essential, for the anesthesiologist, to anticipate respiratory complications (low $\mathrm{SpO}_{2}$, increase in $\mathrm{EtCO}_{2}$, hypoventilation or airway obstruction) and to have a plan of action and the required equipment to deal with difficult airway management. (4)

The anesthesiologist dealing with awake craniotomy has to be aware of the difficulties in predicting the individual right dose of a drug for an awake and anxious patient, as well as respiratory complications due to a post critical state after seizures (table 1).

For successful anesthesia management, accurate selection of the patient as well as clinical examination to detect risks factors, are mandatory. Also, fine tuning of pharmacological titration, accurate monitoring of respiratory rate and expired $\mathrm{CO}_{2}$ and constant free access to the airways are essential for a positive outcome. $(1,2)$

\section{Anesthesia technique: personal experience}

In our institution we routinely perform monitored anesthesia care for awake craniotomy since 1999 ( $n=173$ patients) allowing the patient to breath spontaneously without any tracheal or laryngeal device to protect their airway.

Our standard anesthesia technique includes lorazepam (0.03-0.04 mg/kg) per os the evening before surgery if required. No preoperative sedation is dispensed on the day of surgery. When entering the operating room, all patients receive midazolam 0.03-0.04 $\mathrm{mg} / \mathrm{kg}$ and clonidine $1-1.5 \mu / \mathrm{kg}$ before positioning intravenous and intra arterial catheters. Routine monitoring in the operating room includes: ECG 3 lead system, SpO2, invasive blood pressure, end-tidal carbon dioxide, respiratory rate and hourly urinary output. Oxygen is administrated via a nasal catheter based on $\mathrm{SpO} 2$ value.

Benzodiazepine administration could be criticized, because their effect on vigilance is unpredictable and it could interfere with functional brain mapping. This issue is not confirmed by our experience, bearing in mind that interval elapse between benzodiazepine administration and electrophysiologic mapping of cortical areas (when the patient has to be awake) is at least 2 hours.

The patient receives additional i.v. ranitidine (50 mg), ondansetron (4 mg) and dexamethasone $(0.25 \mathrm{mg} / \mathrm{kg})$. Medications used to treat coexisting illness are kept maintained until surgery.

Once hemodynamic, respiratory and neurological monitoring has been completed, sedation is deepened with remifentanil $(0.02-0.1 \mu \mathrm{g} / \mathrm{kg} / \mathrm{min})$ to obtain an adequate level of analge- sia and sedation. The surgical field, supraorbital, auricular, temporal and occipital nerves are infiltrated with naropine $0.75 \%$ or with a mixture of bupivacaine $0.5 \%$ and mepivacaine $2 \%$ (up to $30 \mathrm{ml}$ ). Throughout the operation, contact between patient, neurosurgeon and anesthesiologist is maintained constantly; the physicians will inform the patients about all the phases of the operation and the patient will communicate any discomfort to the physicians. During patient positioning, it is strongly recommended to avoid an extreme torsion of the cervical spine. At any time during the procedure when excessive pain is expected, such as pterional surgical approach for temporoinsular lesions or for large tumors, additional anesthesia is given by starting with a propofol bolus of $0.5 / 1 \mathrm{mg} / \mathrm{kg}$, followed by a continuous infusion with a rate of 1.5-2 mg/kg/h, aiming to effect-controlled titration; remifentanil infusion is maintained at lower doses. During the surgical procedure some additional medication may be necessary, like rapid infusion of mannitol $(0.5 \mathrm{~g} / \mathrm{kg})$, urapidil or labetalol to control blood pressures. Hypotension (mean arterial pressure $<60 \mathrm{mmHg}$ ) is treated with rapid infusion of colloids or crystalloids and with ephedrine. During awake patient electrical stimulation, temporary loss of neurophysiological function is possible. It must be stressed to the patient that a sudden loss of ability to speak or an appearance of a motor block is a temporary functional disorder and not a permanent one. In our daily practice, we don't use propofol

Table 1. Complications in awake craniotomy. $\mathrm{nr}=$ not reported, $\mathrm{a}=$ retrospective review remifentanil, midazolam and clonidine; $b=$ retrospective review propofol infusion; discontinuation for brain mapping and Ecog; $c=$ retrospective review fentanyl, droperidol or midazolam, propofol infusion; $d=$ prospective trial propofol remifentanil and clonidine; $e=$ prospective trial, 2 groups in spontaneous breathing propofol vs fentanyl and DBP; $f=$ prospective trial, 2 groups fentanyl and propofol vs remifentanil and propofol.

\begin{tabular}{|c|c|c|c|c|c|c|}
\hline Study & Saltarini/Zorzia & Skucas $^{b}$ & Sarangc & Berkenstadt $^{d}$ & Herricke $^{\mathrm{e}}$ & Manninen $^{f}$ \\
\hline Patients $\mathrm{N}^{\circ}$ & 173 & 332 & 99 & 25 & 37 & 50 \\
\hline Airway obstruction \% & 0.5 & 1.8 & 7 & 4 & $5 / 0$ & 18 \\
\hline Hypoxemia SpO2<90\% & 1.7 & 1.5 & $\mathrm{nr}$ & $\mathrm{nr}$ & $\mathrm{nr}$ & $\mathrm{nr}$ \\
\hline Hypercapnia \% & 5.7 & $\mathrm{nr}$ & $\mathrm{nr}$ & $\mathrm{nr}$ & $\mathrm{nr}$ & $\mathrm{nr}$ \\
\hline
\end{tabular}


Table 2. Incidence of complications in awake craniotomy. $a=2$ groups remifentanil vs remifentanil and propofol $\mathbf{g}=$ prospective trial $\mathbf{n = 3 0} \mathbf{3}$ groups fentanyl vs sufentanil vs alfentani. Other symbols as in Table1.

\begin{tabular}{lllllllll} 
Study & $\begin{array}{l}\text { Saltarini } \\
\text { Zorzi }\end{array}$ & $\begin{array}{l}\text { Saltarini } \\
\text { Zorzi }^{\mathrm{a}}\end{array}$ & Skucas $^{\mathrm{b}}$ & Sarang $^{\mathrm{c}}$ & Berkenstadt $^{\mathrm{d}}$ & Herrick $^{\mathrm{e}}$ & Manninen $^{\mathrm{f}}$ & Gignac \\
\hline Nausea \% & 0.8 & 5.8 & 0.9 & $\mathrm{nr}$ & 0 & $10-18$ & 0 & $50-30-70$ \\
\hline Seizures \% & 11 & 8.7 & 3 & 0 & 8 & $0-41$ & $0 / 16$ & $10-30-10$ \\
\hline
\end{tabular}

infusion after the mapping procedure. We made this decision to avoid interference with further electrocortical stimulation, if required by the neurosurgeon. Remifentanil infusion is constantly maintained until the closure of the dura mater, muscular fascia and skin. To improve patient comfort we use low doses of midazolam or continuous infusion of clonidine $(0.3 \mu / \mathrm{min})$. At the end of surgery, the patient receives long acting opioids (morphine $0.1 \mathrm{mg} / \mathrm{kg}$ ) and i.v. paracetamol. If surgery didn't incur any complications, the patient will be monitored in the recovery room for $60-90$ minutes and discharged to the ward as usual. $(14,17)$

\section{Complications}

Complications of awake craniotomies include seizures, swelling of the brain, nausea and vomiting, decreased level of consciousness, neurological deficit, pain and loss of patient cooperation.

Onset of new seizures is a common presentation for a patient with a brain tumor and they may occur unexpected at any time. Most intraoperative seizures may occur during brain mapping (5-20\% of cases), as a result of decreased levels of anticonvulsants, or as a result of local anesthetic toxicity. These seizures may be focal or general and are usually self-limiting. Seizures that occur after rigid head fixation has been applied are particularly frightening. $(6,18,19)$

In our experience we report intraopera- tive seizures in $11 \%$ of cases. Therapy included cortical irrigation with cold ringer's lactate or small doses of propofol or benzodiazepines (diazepam 0.06 $\mathrm{mg} / \mathrm{kg}$ or midazolam $0.04 \mathrm{mg} / \mathrm{kg}$ ). During the post-ictal stage, if airway control is in jeopardy, tracheal intubation may be theoretically necessary: in our study five patients experienced generalized seizures that required conversion to general anesthesia.

The incidence of complications we report with our anesthesia technique is in accordance with those reported in other studies (table 2). Brain swelling was never a problem; other studies reported an incidence of tight brain of $1.4 \%$ (Costello and Archer studies) and $0.6 \%$ in Skucas work.

The incidence of nausea and vomiting is variable, depending on the patient's history, type of lesion, medications, and anesthesia (table 2). Surgical manipulation during dural stripping, temporal lobe or amygdale manipulation, meningeal vessels handling, inadequate analgesia and hypovolemia may all contribute to nausea and vomiting. It can be minimized by a judicious selection of anesthetic drugs combined with the administration of steroids and specific anti-emetic medication. $(6,19)$

Finally, another complication could be the patient's refusal to cooperate. Such events may be due to different reasons including bad preoperative preparation, inappropriate and excessive sedation, insufficient analgesia, uncomfortable position and a too long surgical procedure. Some of them can be prevented, but the loss of patient cooperation, imposes conversion to general anesthesia for safe conclusion of surgery. We never stop to point out that complications may have disastrous consequences and should be prevented rather than treated.

\section{Conclusions}

Neurosurgery in awake patients is an exciting challenge and has become common practice in many surgical centers. The awake craniotomy for tumor surgery has become a well-established procedure, although the anesthetic management will continue to differ for each institution. Our experience is in accordance with data in the literature, which reports this procedure as feasible and safe. New technologies are appearing on the horizon such as infrared cameras to detect functionally active areas of cortex during operations, as well as other established advanced techniques of imaging to guide surgeons and allow complex operations to be performed faster and with fewer complications.

But in spite of all of these technological conquests, the ability and the skill of the anesthesiologist to set up the right pharmacological titration as well as its sensitivity to maintain close psycho-emotional contact with the patient throughout the entire operation will still remain one of the key challenges for a successful outcome. 


\section{REFERENCES}

1. Hans P, Bonhomme V. Anaesthetic management for neurosurgery in awake patients. Minerva Anestesiol 2007;73:507-12.

2. Blanshard HJ, Chung F, Manninen PH. Awake craniotomy for removal of intracranial tumour: consideration of early discharge. Anesth Analg 2001;92:89-4.

3. Manninen PH, Balki M, Lukitto K. Patient satisfaction with awake craniotomy for tumour surgery: a comparison of remifentanil and fentanyl in conjunction with propofol. Anesth Analg 2006;102:237-2.

4. Berkenstadt H, Ram Z. Monitored Anaesthesia Care in awake craniotomy for brain tumour surgery. Isr Med Assoc J 2001;3:297-0.

5. Picht T, Kombos T, Gramm HJ. Multimodal protocol for awake craniotomy in language cortex tumour surgery. Acta Neurochir (Wien) 2006;148:127-38.

6. Costello TG, Cormack JR. Anaesthesia for awake craniotomy: a modern approach. J Clin Neurosci 2004;11(1):16-9.

7. Sarang A, Dinsmore J. Anaesthesia for awake craniotomy evolution of a technique that facilitates awake neurological testing. Br $\mathrm{J}$ Anaesth 2003;90(2):161-5.

8. Berkenstadt H, Perel A, Hadani M. Monitored anaesthesia care using remifentanil and propofol for awake craniotomy. J Neurosurg Anesthesiol 2001;13:246-9.

9. Saltarini M, Zorzi F. Awake craniotomy Minerva Anestesiol 2005;71:183-5.

10. Johnson KB, Egan TD. Remifentanil and propofol combination for awake craniotomy: case report with pharmacokinetic simulations. J Neurosurg Anesthesiol 1998;10:25-9.

11. Hans P, Bonhomme V, Born JD, Maertens de Noordhoudt A, Brichant JF, Dewandre PY. Target-controlled infusion of propofol and remifentanil combined with bispetral index monitoring for awake craniotomy. Anaesthesia 2000;55:255-9.

12. Skucas AP, Artru AA. Anaesthetic complications of awake craniotomies for epilepsy surgery. Anesth Analg 2006;102:882-7.

13. Yamamoto F, Kato R, Sato J. anaesthesia for awake craniotomy with non-invasive pressure ventilation. Br J Anaesth 2003;90:382-5

14. Saltarini M, Zorzi F, Bonassin P. Awake craniotomy: back to the future. NeuroAnesth Crit Care Siena 2005;139-52.

15. Bekker AY, Kaufman B, Samir H, Doyle W. The use of dexmedetomidine infusion for awake craniotomy. Anesth Analg 2001;92(5):1251-3.

16. Moore TA, Markert JM, Knowlton RC. Dexmedetomidine as rescue drug during awake craniotomy for cortical motor mapping and tumour resection. Anesth Analg 2006;102:1556-8.

17. Saltarini M, Zorzi F. Awake Anestesia: i rischi per il paziente e per l'anestesista. NeuroAnesth Crit Care Siena 2007. In press.

18. Sartorius CJ, Berger MS. Rapid termination of intraoperative stimulation - evoked seizures with application of cold Ringer's lactate to the cortex. J Neurosurg 1998;88:349-35.

19. Sartorius CJ, Wrigth G. Intraoperative brain mapping in a community setting-technical considerations. Surg Neurol 1997;47:380-8. 